\title{
Changes in Striatal Dopamine Neurotransmission Assessed with Microdialysis Following Recovery from a Bilateral 6-OHDA Lesion: Variation as a Function of Lesion Size
}

\author{
Edward Castañeda, ${ }^{1}$ lan Q. Whishaw, ${ }^{1}$ and Terry E. Robinson ${ }^{2}$ \\ 'Department of Psychology, University of Lethbridge, Lethbridge, Alberta, Canada TIK 3M4, and ${ }^{2}$ Department of \\ Psychology and Neuroscience Program, The University of Michigan, Ann Arbor, Michigan 48109
}

Intracerebral microdialysis was used to measure the extracellular concentration of striatal dopamine (DA) and its metabolites in freely moving rats depleted of DA by the bilateral infusion of 6-hydroxydopamine into the substantia nigra approximately 1 month earlier. It was found that the basal extracellular concentration of DA remained within the same range as seen in control animals until the size of the lesion exceeded $80 \%$ (estimated by the postmortem tissue concentration of DA). In animals with an $80-95 \%$ lesion there was only a modest decrease in basal extracellular DA, but as lesion size exceeded $95 \%$ there was a marked drop in the basal extracellular concentration of DA. In contrast, the basal extracellular concentration of the DA metabolites showed a more steady decline as a function of lesion size. To determine the ability of the residual population of DA terminals to further increase DA release upon increased demand, animals were given a challenge injection of $1.5 \mathrm{mg} /$ $\mathrm{kg}$ of $d$-amphetamine. Amphetamine-evoked DA release remained within the control range until lesion size exceeded $95 \%$. These results provide direct confirmation for the hypothesis that following recovery from partial bilateral damage to the nigrostriatal DA system in adulthood, there are presynaptic compensatory changes in the remaining population of DA neurons sufficient to "normalize" the extracelJular concentration of striatal DA. It is suggested that this normalization of extracellular DA is responsible for the sparing of function seen following the loss of up to $80 \%$ of the DA innervation to the striatum and contributes to the recovery of function seen after even more extensive damage (80$95 \%)$. However, at least $5 \%$ of the usual dopaminergic innervation to the striatum is required to maintain a relatively normal extracellular concentration of DA, and it appears that this $\mathbf{5} \%$ is necessary for any significant recovery of function.

The bilateral destruction of dopamine (DA)-containing neurons in the substantia nigra with a neurotoxin such as 6-hydroxydopamine (6-OHDA) or 1-methyl-4-phenyl-1,2,3,6-tetrahydropyridine (MPTP) causes a widespread depletion of DA throughout the primary projection field of these cells in the neostriatum

\footnotetext{
Received Oct. 19, 1989; revised Jan. 18, 1990; accepted Jan. 25, 1990.

This research was supported by grants from the MRC and NSERC of Canada to I.Q.W. and from the U.S. Public Health Service to T.E.R. Dr. Castañeda is an Alberta Heritage Foundation Fellow at the University of Lethbridge.

Correspondence should be addressed to Dr. Edward Castañeda at the above address.

Copyright (C) 1990 Society for Neuroscience $0270-6474 / 90 / 061847-08 \$ 02.00 / 0$
}

(Ungerstedt, 1968; Heikkila et al., 1984). The depletion of striatal DA in animals results in the appearance of a behavioral syndrome that is considered an animal analog of Parkinson's disease in humans (Schultz, 1982). In rats this is characterized by bradykinesia, sensorimotor neglect, aphagia, adipsia, akathisia, short-step locomotion, postural abnormalities, and cognitive dysfunction (Ungerstedt, 1971; Zigmond and Stricker, 1972; Marshall et al., 1974; Stricker and Zigmond, 1974; Zis et al., 1974; Schallert and Whishaw, 1978; Schallert et al., 1978; Whishaw et al., 1978, 1986; Whishaw and Dunnett, 1985).

The severity of behavioral deficits following DA depletion is not related to lesion size in a simple way. If essentially the entire DA input to the striatum is destroyed (>95-98\%), animals show a permanent loss of function. For example, they may never reacquire spontaneous ingestive behavior and will show bradykinesia and sensorimotor neglect for as long as they are kept alive (Zigmond and Stricker, 1973; Marshall, 1979). If the lesion destroys only from 80 to $95 \%$ of the DA innervation to the striatum, animals initially show the behavioral syndrome described above, but if kept alive by tube feeding, many eventually will recover their former behavioral capacities (Zigmond and Stricker, 1973; Marshall and Teitelbaum, 1974; Marshall, 1979). The process of recovery of function seen following DA depletion is similar to that described earlier in animals with lateral hypothalamic lesions (Teitelbaum and Epstein, 1962; Ungerstedt, 1971; Marshall ct al., 1974; Robinson and Whishaw, 1974). Interestingly, the more salient behavioral deficits produced by DA depletion are not seen if striatal DA is depleted by less than about $80 \%$; that is, there is sparing of function (Stricker and Zigmond, 1976). This nonlinear relation between extent of depletion and behavioral deficits is also similar to Parkinson's disease, in which clinical symptoms are typically not manifest until over $80 \%$ of the DA input to the striatum is lust (Hornykiewicz, 1972). It appears, therefore, that only $20 \%$ of the normal dopaminergic input to the striatum is required to maintain relatively normal function.

These neurochemical and behavioral observations have led to considerable interest in the neuronal adaptations responsible for recovery and sparing of function following DA depletion. It has been hypothesized that following moderate damage to the nigrostriatal DA system there are changes in the synthesis, metabolism, and release of DA from the remaining terminals that are sufficient to maintain a higher extracellular (synaptic) concentration of DA than would be predicted by the amount of cell loss or the depletion of DA in postmortem tissue (Stricker and Zigmond, 1976, 1986). Following a large lesion $(>80-90 \%)$ these 
presynaptic changes presumably interact with supersensitive postsynaptic DA receptors to mediate recovery of function. This hypothesis has not been tested directly in animals depleted of DA in adulthood, however, because the measures of biosynthesis and metabolism used in previous studies provided only indirect (and sometimes unreliable) measures of extracellular DA (Commissiong, 1985; Westerink, 1985).

We recently used the technique of microdialysis to directly measure the extracellular concentration of DA in the striatum of freely moving rats that were either treated neonatally with 6-OHDA (Castañeda et al., 1990) or given a unilateral 6-OHDA lesion of the substantia nigra in adulthood (Robinson and Whishaw, 1988). It was found that the extracellular concentration of DA in the striatum of these animals was much higher than could be predicted by the amount of DA depletion in postmortem tissue, and it was suggested that this "normalization" of extracellular DA may contribute to sparing and recovery of function. The present experiment was designed to determine if a similar normalization of extracellular DA occurs following recovery from bilateral DA depletion produced by an injection of 6-OHDA into the substantia nigra during adulthood. Furthermore, the size of the lesion (amount of DA depletion) was varied to determine the limits of presynaptic compensatory changes that serve to maintain the extracellular concentration of DA; and animals were given a challenge injection of $d$-amphetamine to determine their ability to respond to an increased demand for DA release.

\section{Materials and Methods}

Subjects. Adult male rats (Holtzman, $n=22$, or Long-Evans, $n=22$ ), weighing 232-682 gm, were housed on a 14:10 hr light : dark cycle (lights on at 0800 ) with free access to food and water. Animals in the lesion group $(n=30)$ received bilateral infusions of $6-\mathrm{OHDA} \cdot \mathrm{HBr}$ into the rostral zona compacta of the substantia nigra using procedures described previously (Robinson and Whishaw, 1988). Briefly, animals were pretreated with $25 \mathrm{mg} / \mathrm{kg}$ of desipramine $\mathrm{HCl}$ (i.p.) at least $30 \mathrm{~min}$ prior to the 6-OHDA infusion (Breese and Traylor, 1971) and then were anesthetized with sodium pentobarbital. Each animal received 3-8 $\mu \mathrm{g}$ of $6-\mathrm{OHDA} \cdot \mathrm{HBr}$ in $4 \mu \mathrm{l}$ of vehicle $(0.9 \% \mathrm{NaCl}$ solution containing 0.1 $\mathrm{mg} / \mathrm{ml}$ ascorbate) into each substantia nigra via a $30 \mathrm{~g}$ stainless steel cannula (coordinates from bregma: posterior, $5.0 \mathrm{~mm}$; lateral, $2.0 \mathrm{~mm}$; ventral, $7.4 \mathrm{mIn}$; with bregma and lambda horizontal). The animals were then returned to their home cages and allowed to recover for at least 1 month before testing began. Animals that were aphagic and adipsic following surgery were tube-fed until they recovered sufficiently to maintain their body weight, or until dialysis began. Control animals ( $n=14)$ were treated the same as described above, but the infusion cannulae were not lowered.

On the day prior to microdialysis testing the animals were again anesthetized with sodium pentobarbital $(30-35 \mathrm{mg} / \mathrm{kg}$, i.p.) and a dialysis probe was stereotaxically placed into the corpus of the striatum on either the left or right side, with the side randomly chosen (coordinates from bregma: anterior, $0.5 \mathrm{~mm}$; lateral, $2.5 \mathrm{~mm}$; ventral, 7.0 $\mathrm{mm}$; with bregma and lambda horizontal). The probe was fixed in place with dental cement and jeweler's screws attached to the skull. The animals were then placed in the test chamber and left overnight. Testing began the next day between 0800 and $1000 \mathrm{hr}$, at least $18 \mathrm{hr}$ after probe implantation.

Microdialysis procedures. The dialysis procedure and probe design have been described in detail previously (Robinson and Whishaw, 1988; Robinson et al., 1988; Castañeda et al., 1990). Briefly, the dialysis probe was of a concentric design with an outer diameter of $250 \mu \mathrm{m}$ and a 4-mm-long tip. The outlet line consisted of fused silica capillary tubing (I. D., $75 \mu \mathrm{m}$ ) which led to a collection vial attached to the tether, about $35 \mathrm{~cm}$ above the animal. Thus, collection vials could be quickly exchanged without touching the animal or impeding its movement.

On the morning of a test day the pump speed was set at $1.5 \mu \mathrm{l} / \mathrm{min}$ and sample collection began 30 min later. Baseline samples were typically collected over $20 \mathrm{~min}$ intervals, but samples were collected for up to $50 \mathrm{~min}$ in animals with low basal levels of DA, providing a greater volume of dialysate for assay. Samples were assayed within $30 \mathrm{~min}$ of collection using HPLC and series oxidative-reductive electrochemical detection, as described previously (Castañeda et al., 1990). After baseline samples were collected and assayed, the animals were given a subcutaneous injection of $1.5 \mathrm{mg} / \mathrm{kg}$ of $d$-amphetamine sulfate (weight of the salt) in the nape of the neck, and 10 more samples were collected over 20 min intervals.

The animals were videotaped during testing and their behavior subsequently analyzed by an observer blind to the treatment conditions. The videotapes were viewed at 2 times normal speed, and the number of rearing movements (both front feet off the floor) and the number of times the midline of the box was crossed (crossovers) were tabulated over $10 \mathrm{~min}$ intervals.

At the end of the experiment the tubing leading to the dialysis probe was severed at the level of the dental cement "cap," the open ends were sealed with epoxy, and the animal was returned to its home cage. Approximately $5 \mathrm{~d}$ later each animal was killed by decapitation and its brain rapidly removed and placed in ice-cold saline. After the brain cooled (30-45 sec) it was placed in a chilled cutting block and brain slices were obtained (Heffner et al., 1980). The slices were immediately examined and the approximate location of the "track" formed by the dialysis probe was reconstructed. The left and right striatum and nucleus accumbens were then dissected, weighed, and placed into individual tubes containing $0.05 \mathrm{~N}$ perchloric acid and dihydroxybenzylamine (internal standard). The samples were homogenized and centrifuged at $1500 \times g$ for $4 \mathrm{~min}$. The supernatant was assayed by HPLC and electrochemical detection, using procedures similar to those described previously (Robinson et al., 1987).

All probes were tested for recovery in vitro prior to use (Robinson and Whishaw, 1988) and all dialysate values were corrected for recovery. The average $( \pm$ SEM) relative recovery values for the probes used in this experiment were: DA, $25.44 \pm 0.40 \%$; dihydroxyphenylacetic acid (DOPAC), $23.43+0.38 \%$; homovanillic acid (HVA), $22.46+0.34 \%$; and 5-hydroxyindoleacetic acid (5-HIAA), $25.05 \pm 0.52 \%$.

\section{Results}

Basal extracellular concentrations of $D A, D O P A C$, and $H V A$ Figure 1 shows the basal (resting) extracellular concentration of DA, DOPAC, and HVA in every single control and 6-OHDAtreated animal, plotted as a function of lesion size (\% DA depletion). The size of the lesion was estimated by determining the degree of DA depletion in postmortem tissue obtained from the striatum of each 6-OHDA-treated animal (on the dialyzed side) relative to the average postmortem tissue concentration of striatal DA in control animals [i.e., (Control - lesion)/control $\times 100]$. A number of studies have shown that the postmortem tissue concentration of DA provides a very good index of the density of dopaminergic innervation in rats with a 6-OHDA lesion (Ranje and Ungerstedt, 1977; Onn et al., 1986; Altar et al., 1987).

It can be seen in Figure $1 A$ that the basal extracellular concentration of DA remained within the control range (18.6 to 62 $\mathrm{fmol} / \mathrm{min}$; mean $\pm \mathrm{SEM}$ of $37 \pm 3.7)$ in most rats with a 6-OHDA lesion until the size of the lesion started to exceed about $80 \%$. Even most rats with an $80-95 \%$ lesion maintained basal extracellular DA within the control range, although all values were below the control average. As lesion size exceeded $95 \%$, there was a sharp decline in the extracellular concentration of striatal DA to barely detectable levels. The relationship between the extracellular concentration of DA and lesion size is also illustrated in Figure $2 A$, in which 6-OHDA-treated animals have been subdivided into 3 subgroups, those with: (1) $20-80 \%$ depletion in the postmortem tissue concentration of DA; (2) $80-95 \%$ depletion; and (3) $>95 \%$ depletion (see Table 1). There was no statistically significant difference in the basal extracellular concentration of DA in control animals and animals with a $20-80 \%$ lesion. In animals with an $80-95 \%$ lesion basal ex- 


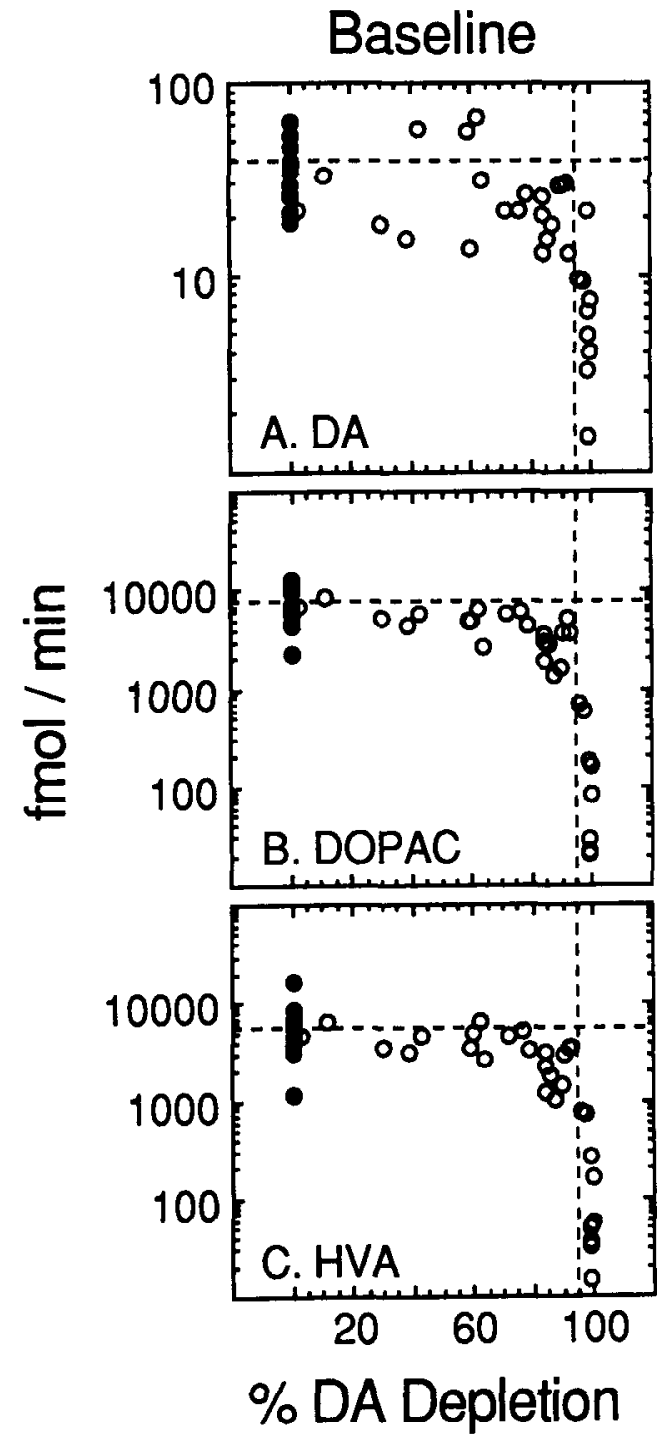

Figure 1. Resting (basal) extracellular concentration of dopamine ( $A$. $D A)$, dihydroxyphenylacetic acid ( $B . D O P A C)$, and homovanillic acid (C. $H V A$ ), expressed in $\mathrm{fmol} / \mathrm{min}$ (corrected for recovery), in individual control animals (closed symbols; $n=14$ ) and animals treated with 6-OHDA (open symbols; $n=30$ ), and plotted as a function of the percent DA depletion in postmortem tissue. Note the log scale on the ordinate. Percent DA depletion was determined by comparison of the postmortem tissue concentration of DA in the striatum on the dialyzed side of each 6-OHDA-treated animal with the average concentration of DA in postmortem tissue from control animals (control - lesion/control $\times 100$ ). The horizontal dashed line represents the average extracellular concentration of DA in control animals and the vertical dashed line the point of $>95 \%$ lesion.

tracellular DA was significantly less than in controls (Fig. $2 A$ ), and in animals with a $>95 \%$ lesion extracellular DA was greatly reduced relative to all other groups.

Although the basal extracellular concentration of DA was reduced by a large 6-OHDA lesion, the rate of DA release from the remaining DA terminals greatly increased with increasing lesion sizc, as indicated by the estimate of fractional DA release shown in Figure 3 (i.e., extracellular DA calculated as a percent of total DA in postmortem tissue).

In this experiment approximately half the control and lesion animals were tested at the University of Michigan (Holtzman)
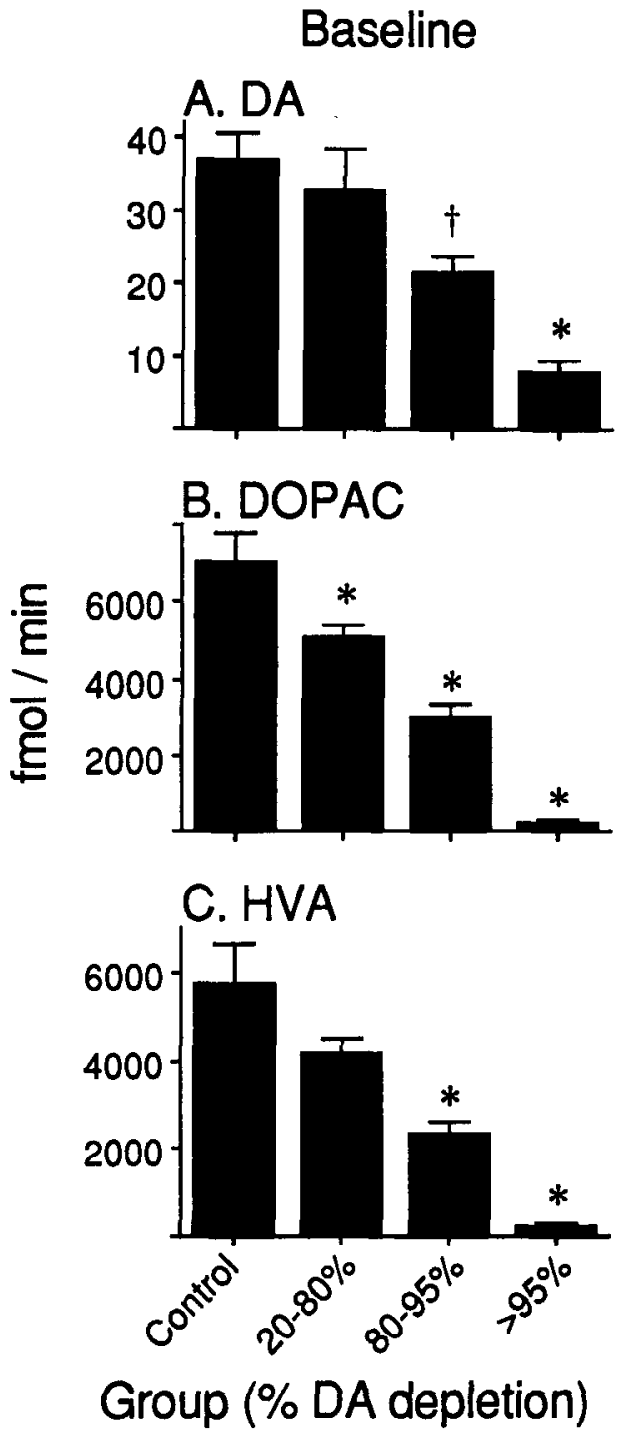

Figure 2. Average (+SEM) extracellular concentration of DA $(A)$, DOPAC $(B)$, and HVA $(C)$ during baseline expressed in $\mathrm{fmol} / \mathrm{min}$ (corrected for recovery) for the same animals as shown in Figure 1, but grouped into 4 subgroups: (1) control $(n=14)$; (2) 6-OHDA-treated with a $20-$ $80 \%$ DA depletion (mean depletion $=58.1 \% ; n=10$ ) in postmortem tissue; (3) 6-OHDA-treated with an 80-95\% DA depletion (mean $=$ $87.8 \% ; n=9$ ); and (4) 6-OHDA-treated with a $>95 \%$ DA depletion (mean $=98.3 \% ; n=9$ ). Analyses of variance resulted in a significant effect of group (lesion size) for all 3 compounds ( $F \mathrm{~s}=10.1,23.7$, and 12.4 for DA, DOPAC, and HVA, respectively; $p<0.001$ ). The asterisks $\left({ }^{*}\right)$ indicate groups that differed significantly from all other groups (including control) and the dagger $(\dagger)$ groups that differed from control (planned 2-tailed $t$-tests, $p<0.05$ ).

and half at the University of Lethbridge (Long-Evans), and some of the considerable variability in the extracellular concentration of DA seen even in control animals may be due to strain differences and/or differences in the testing environment at the 2 locations. There was a significant strain difference in the basal extracellular concentration of DA $(t=2.23, p=0.046)$, but not in the peak amphetamine-evoked release of DA $(t<1.0)$. Nevertheless, exactly the same pattern of results was seen when the 2 strains were analyzed separately, and therefore, all animals were pooled for the analyses reported here (although this probably resulted in somewhat greater variability than normal). 


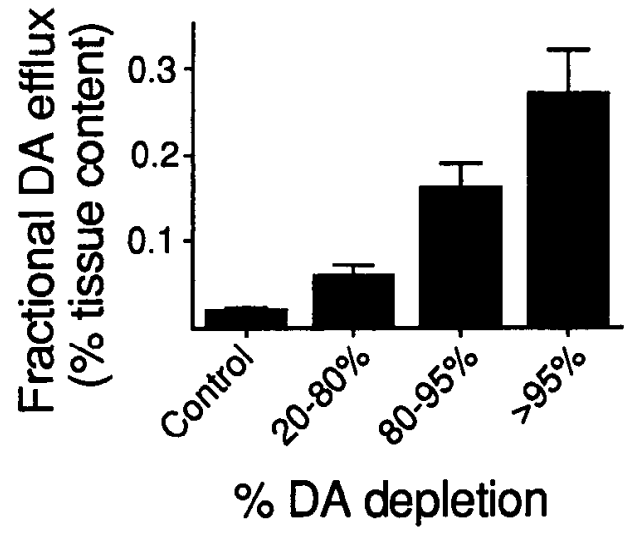

Figure 3. Fractional rate of DA efflux during baseline in each of the subgroups described in Figure 2. Fractional DA release was calculated by expressing the extracellular concentration of DA $(\mathrm{pg} / \mu \mathrm{l})$ as a percent of the postmortem tissue concentration of DA $(\mathrm{pg} / \mathrm{mg})$. All groups differed significantly from all other groups [analysis of variance (ANOVA) and $t$-tests].

The basal extracellular concentrations of DOPAC and HVA were reduced by the 6-OHDA lesion (Fig. 1, B, C). In contrast to DA, the basal extracellular concentration of DOPAC and HVA was below the control average in nearly all animals with a $>20 \%$ lesion, although values tended to remain within the control range until the lesion exceeded about $80 \%$. The steady decline in extracellular DOPAC and HVA as a function of lesion size is evident in Figure 2, $B, C$, in which average values for the 3 subgroups are shown.

\section{Effects of an amphetamine challenge on the extracellular concentration of $D A, D O P A C$, and IIVA}

Animals were given a challenge injection of $d$-amphetamine (1.5 $\mathrm{mg} / \mathrm{kg}$ ) to determine their ability to enhance DA rclcase in response to increased demand. Figure $4 A$ shows the average extracellular concentration of DA in every animal during the first three 20-min intervals following amphetamine administration, when DA levels were the highest (peak DA; see Fig. $5 A$ ), and plotted as a function of lesion size. Animals with a 6-OHDA lesion increased extracellular DA to the same extent as control animals until lesion size started to exceed about $95 \%$. Figure $4 B$ shows the average concentration of extracellular DA in each of the subgroups over the entire 200 min following amphetamine administration, and again, amphetamine-stimulated DA release was significantly depressed only in animals with a $>95 \%$ lesion. There was a tendency for animals with an 80-95\% lesion to release less DA than controls, but this was not statistically significant. The full time course of the DA response to the amphetamine challenge is illustrated in Figure $5 \mathrm{~A}$.

It is interesting to compare the change in extracellular DA produced by the amphetamine challenge with the behavioral response to the amphetamine challenge. Figure $4 C$ shows the total number of crosses plus rears during the 200 min test session following amphetamine administration in control animals and in each of the 6-OHDA subgroups. Animals with a $>95 \%$ lesion showed significantly less behavioral activation than did all other groups, including controls, in association with the reduction in DA release (Fig. $4 B$ ). In contrast, animals with an $80-95 \%$ lesion showed a significantly greater behavioral response to the amphetamine challenge than either controls or animals with a $>95 \%$ lesion, despite the absence of a significant change in extracellular DA (Fig. 4B). Animals with a $20-80 \%$ lesion did not differ significantly from control.

Figure 5, $B$ and $C$, shows the extracellular concentrations of DOPAC and HVA during baseline and at each of the ten 20min intervals following the amphetamine challenge. Amphetamine produced a decrease in the extracellular concentrations of DOPAC and HVA in all groups, although the large lesioninduced depletion of DOPAC and HVA is evident in all three 6-OHDA-treated subgroups and at all points in time.

\section{5-HIAA}

Figure $6 \mathrm{~A}$ shows the basal extracellular concentration of 5-HIAA in all animals, plotted as a function of lesion size, and Figure $6 B$ illustrates the effect of the amphetamine challenge on 5-HIAA in control animals and each of the 6-OHDA subgroups. It is obvious that there was no effect of 6-OHDA on the basal extracellular concentration of 5-HIAA, regardless of lesion size. The amphetamine challenge did produce a small, but statistically significant change in $5-\mathrm{HI} \Lambda \Lambda$ over time, characterized by a transient decline followed by a steady increase-but there were no significant group differences.

\section{Discussion}

The results reported here provide the first direct confirmation for the hypothesis that following recovery from partial bilateral damage to the nigrostriatal DA system in adulthood there are presynaptic compensatory changes in the remaining population of DA neurons that are sufficient to maintain a relatively normal extracellular concentration of DA in the striatum (Stricker and Zigmond, 1976, 1986). The exact nature of the presynaptic ad-

Table 1. Mean ( \pm SEM) concentration of dopamine (ng $/ \mathrm{mg}$ wet tissue weight) in postmortem tissue from the striatum (caudate putamen) and the nucleus accumbens of control animals and 3 subgroups of animals treated with 6-OHDA

\begin{tabular}{llccc} 
& & \multicolumn{3}{l}{ 6-OHDA (\% DA depletion) } \\
\cline { 2 - 4 } & Control & $20-80$ & $80-95$ & $>95$ \\
\hline Striatum & $17.81 \pm 1.88$ & $6.93 \pm 1.14$ & $1.91 \pm 0.35$ & $0.33 \pm 0.09$ \\
(\% depletion) & & $(58.1 \pm 4.9)$ & $(87.8 \pm 1.1)$ & $(98.3 \pm 0.4)$ \\
Nucleus accumbens & $13.35 \pm 1.53$ & $9.77 \pm 1.32$ & $7.76 \pm 1.21$ & $3.58 \pm 1.22$ \\
$(\%$ depletion) & & $(26.8 \pm 9.9)$ & $(41.9 \pm 9.1)$ & $(73.2 \pm 9.2)$ \\
\hline
\end{tabular}

The 6-OHDA subgroups are those with $20-80 \%$ dopamine depletion in postmortem tissue (relative to controls), those with $80-95 \%$ depletion, and those with $>95 \%$ depletion (as described in the text and Fig. 2). The numbers in parentheses represent the mean $( \pm$ SEM) percent depletion for each 6-OHDA subgroup, relative to the controls $(n=9-14$; see Fig. 2). 


\section{A. Amphetamine}

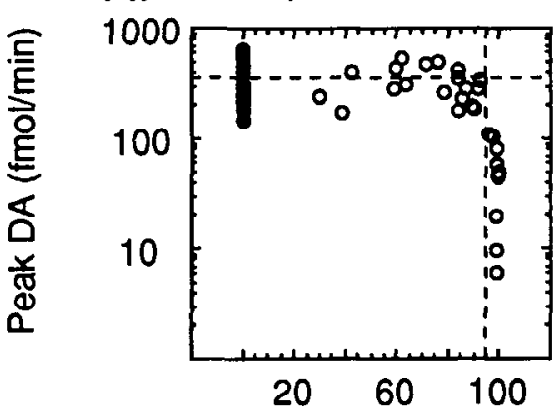

B.

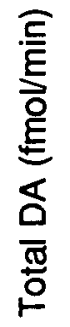

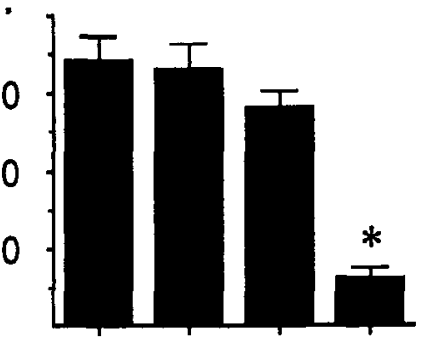

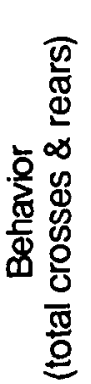

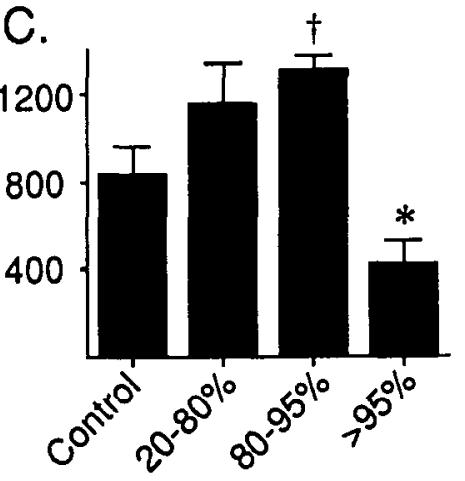

\section{\% DA Depletion}

Figure 4. Effects of $1.5 \mathrm{mg} / \mathrm{kg}$ of $d$-amphetamine on the extracellular concentration of DA and behavior in control and 6-OHDA-treated animals. $A$, Peak extracellular concentration of DA (averaged over the first 320 -min intervals after amphetamine administration) for each individual control and 6-OHDA-treated animal plotted as a function of the \% DA depletion in postmortem tissue (as in Fig. 1). B, Extracellular concentration of DA averaged across the entire 200 -min test session following amphetamine administration in each of the 4 subgroups described in Figure 2. There was a significant effect of group on the extracellular concentration of DA $(F=17.8, p<0.0001)$, and the asterisk $\left({ }^{*}\right)$ indicates that animals with a $>95 \%$ lesion differed significantly from all other groups ( $p<0.001$, relative to controls). The remaining groups did not differ significantly from one another (planned $t$-tests). $C$. Total number of crossovers and rearing movements in each subgroup over the entire 200-min test session after amphetamine administration. There was a significant effect of group $(F=7.2, p<$ $0.001)$, and the asterisk $\left(^{*}\right)$ indicatcs that animals with a $>95 \%$ lesion differed from all other groups $(p<0.04$, relative to controls). The dagger $(\dagger)$ indicates that the $80-95 \%$ lesion group also differec sigrificantly from control $(p<0.01)$.

aptations that culminate in the normalization of extracellular DA is not well understood. A comparison of the fractional release rate in control and 6-OHDA-treated animals certainly suggests that the remaining DA terminals greatly increase their rates of DA release, which is consistent with in vitro studies (Stachowiak et al., 1987).
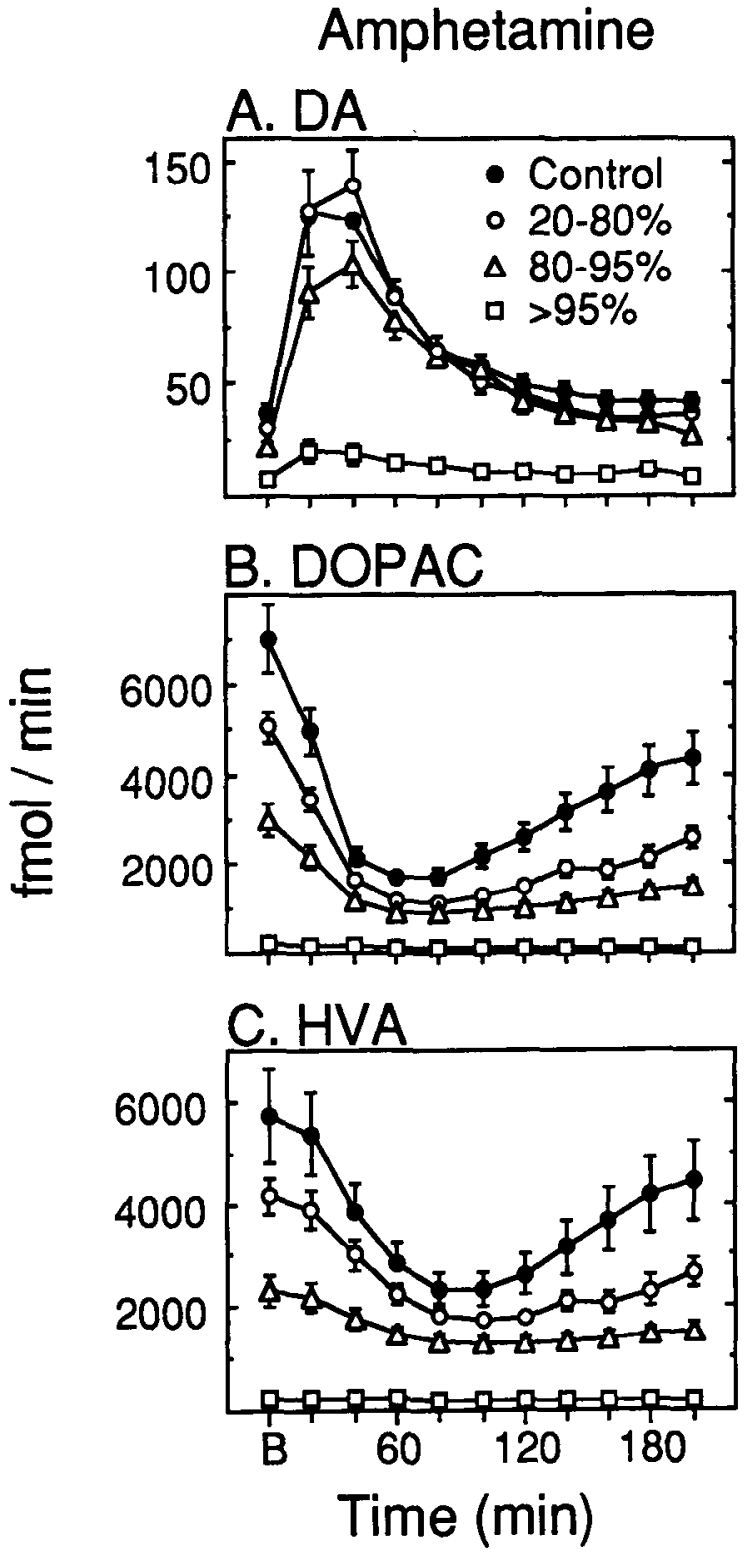

Figure 5. Average ( \pm SEM) extracellular concentration $(\mathrm{fmol} / \mathrm{min})$ of $\mathrm{DA}(A)$, DOPAC $(B)$, and HVA $(C)$ during baseline ( $B$ on the horizontal axis) and for each of 1020 -min intervals following $1.5 \mathrm{mg} / \mathrm{kg}$ of $d$-amphetamine. The 4 groups include controls (solid circles) and 6-OHDA-treated rats with a 20-80\% (open circles), 80-95\% (open iriangles), or $>95 \%$ (open squares) depletion of DA in postmortem tissue (as described in Fig. 2 and the text). For symbols with no SEM bars the SEM is smaller than the symbol. $A$. $D A$, An overall ANOVA comparing all 4 groups across time resulted in a significant effect of group $(F=$ $17.8, p<0.0001)$, lime $(F=68.2, p<0.0001)$, and a group by time interaction $(F=5.5, p<0.0001)$. Follow-up ANOVAs comparing each individual group showed that animals with a $>95 \%$ lesion differed from all other groups (e.g., compared to controls, $F=42.8, p<0.0001$ ), but none of the other 3 groups differed significantly from one another. $B$. $D O P A C$, All groups differed significantly from all other groups (ANOVAs, $F$ 's $=6.7-60.3, p$ 's $<0.02-0.0001) . C$. $H V A$, All groups differed significantly from all other groups (ANOVAs, $F$ s $=7.5-94.7, p$ 's $<$ $0.012-0.0001$ ).

Another lesion-evoked change that has been well characterized, and must be important for the normalization of extracellular DA, is an increase in DA synthesis. Early after a 6-OHDA lesion this is due primarily to a change in the affinity of tyrosine hydroxylase for its cofactor, and later in recovery to an increase 

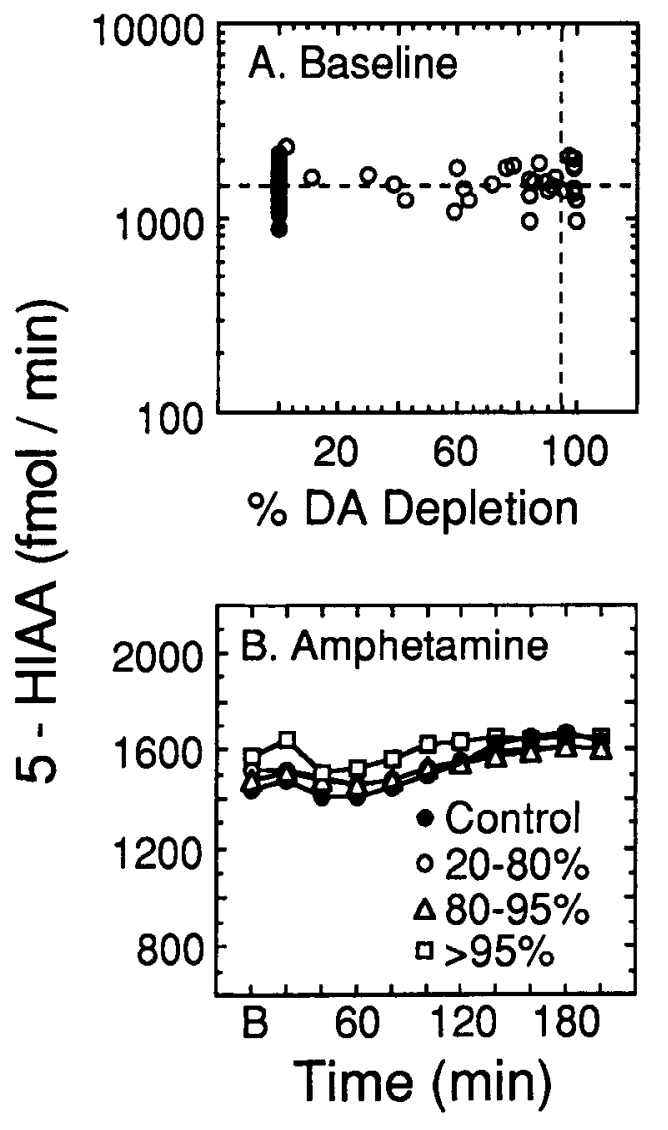

Figure 6. A, Basal extracellular concentration of 5-HIAA in $\mathrm{fmol} / \mathrm{min}$ (corrected for recovery) in individual control animals (solid symbols) and animals treated with 6-OHDA (open symbols) plotted as a function of the $\%$ DA depletion in postmortem tissuc (as described in Fig. 1). $B$, Average extracellular concentration of 5-HIAA during baseline ( $B$ on the horizontal axis) and for each of 1020 -min intervals following the administration of $1.5 \mathrm{mg} / \mathrm{kg}$ of $d$-amphetamine. The 4 groups are the same as in Figure 4. The SEM bars are not shown because all 4 groups overlap at all points in time. An overall ANOVA resulted in a nonsignificant group effect $(F<1.0)$, although there was a significant change in extracellular 5-HIAA over time following amphetamine administration $(F=47, p<0.0001)$. This was characterized by a small initial decline followed by a small gradual elevation in extracellular 5-HIAA, and this effect was somewhat more pronounced in animals with a $>95 \%$ lesion than in the other groups.

in the amount of tyrosine hydroxylase available (Zigmond et al., 1984, 1986; Wolf et al., 1989). This increase in DA synthesis is probably necessary to maintain a high rate of DA release from the surviving DA terminals. The effects of a 6-OIIDA lesion on the rate and pattern of discharge in the remaining population of DA neurons in the substantia nigra have been less well characterized. There are reports that following a 6-OHDA lesion the remaining DA neurons increase their rate of discharge, and that previously "silent" cells become active (Hollerman et al., 1986; Hollerman and Grace, 1988), but how these changes relate to the size of the lesion, the normalization of extracellular DA or behavioral sparing and recovery of function is largely unexplored.

A factor that is probably important for the normalization of extracellular DA, and has not been studied in any detail, is the loss of DA reuptake sites and resultant increase in the diffusibility of DA through the extracellular space. In fact, an increase in the diffusibility of DA may be the single most important change which allows DA released from the remaining terminals to influence distant targets (Doucet et al., 1986) and thus maintain function. All these presynaptic adaptations, and perhaps other as yet unknown neuronal changes, presumably interact to result in the normalization of extracellular DA described here.

Nevertheless, the ability of animals with a 6-OHDA lesion to normalize extracellular DA is dependent on the number of surviving DA terminals. It was found that if $20 \%$ or more of the dopaminergic innervation to the striatum survived, i.e., the lesion destroyed $\angle 80 \%$ of the DA input, the basal extracellular concentration of DA was completely normal. Similar results have been reported recently using 3-methoxytyramine as an index of DA release (Altar and Marien, 1989), with microdialysis in rats with a unilateral 6-OHDA lesion (Robinson and Whishaw, 1987, 1988; Zhang et al., 1988), and with microdialysis in rats that had a bilateral DA depletion induced by neurotoxic doses of methamphetamine (Robinson et al., 1990). Furthermore, it was found here that animals with a $20-80 \%$ lesion showed a completely normal increase in the extracellular concentration of DA in response to a challenge injection of 1.5 $\mathrm{mg} / \mathrm{kg}$ of $d$-amphetamine, indicating that they were able to further elevate DA release to meet demand.

Of course, animals that have over $20 \%$ of the DA input to the striatum intact do not show the typical "DA depletion syndrome" of bradykinesia, aphagia, and sensorimotor neglect; i.c., these functions are spared (Marshall, 1979; Stricker and Zigmond, 1986). It seems highly probable that the normalization of extracellular DA seen here is involved in the sparing of function characteristic of animals with a $<80 \%$ lesion. This normalization of extracellular DA is perhaps also the reason why clinical symptoms do not appear in Parkinson's disease until uver $80 \%$ of the DA content of the striatum is lost. It is unlikely that DA receptor supersensitivity plays an important role in sparing of function because receptor supersensitivity does not develop until over $80 \%$ of the DA input to the striatum is lost and severe behavioral deficits appear (Stricker and Zigmond, 1986).

Animals with only $5-20 \%$ of the usual DA input to the striatum intact were also able to normalize the extracellular concentration of DA to a remarkable extent (Robinson and Whishaw, 1988). Although there was a significant decrease in the basal extracellular concentration of DA in these animals, it was not nearly as great as would be predicted by the extent of the DA depletion in postmortem tissue. DA in postmortem tissue was depleted by an average of $87.8 \%$ in this subgroup, whereas the basal extracellular concentration of DA was reduced by less than half this ( $41.6 \%$, relative to controls). Furthermore, animals with an $80-95 \%$ lesion were able to greatly increase the extracellular concentration of DA in response to an amphetamine challenge, and although on average the magnitude of the response was slightly less than control, it was not significantly less. It is possible that if the demand for increased DA release were even greater, for example, by challenging with a higher dose of amphetamine, these animals would be deficient. Nevertheless, it is clear that only $5-20 \%$ of the normal DA innervation to the striatum is necessary to maintain a relatively high extracellular concentration of $\mathrm{DA}$, and that this small residual population of DA terminals is capable of further increasing DA release upon demand (Robinson and Whishaw, 1988).

Animals with an 80-95\% lesion initially show the full "DA depletion syndrome" described above, and most recover from 
their deficits over time, although the rate and extent of recovery are variable (Marshall, 1979; Stricker and Zigmond, 1986). It is tempting to speculate that the recovery of function associated with an $80-95 \%$ lesion is related to the ability of these animals to maintain a relatively high extracellular concentration of DA. It must be emphasized, however, that in the present study extracellular DA was measured over a month after the 6-OHDA lesion, following the recovery process. The data are consistent with the idea that the normalization of extracellular DA is necessary for recovery of function, but they do not establish whether this is sufficient. That a minimal number of DA terminals are necessary for recovery of function is known from studies showing that if over $95 \%$ of the DA projection field in the striatum is destroyed, the probability that an animal will show recovery of function is greatly diminished, despite pronounced DA receptor supersensitivity (Marshall, 1979; Stricker and Zigmond, 1986). It was found here that a $>95 \%$ lesion produced a marked drop in the basal extracellular concentration of DA, and that these animals were totally unable to increase DA release in response to an amphetamine challenge. It appears, therefore, that at least $5 \%$ of the normal DA input to the striatum is also required to maintain a relatively normal extracellular concentration of DA, and this $5 \%$ must be critical for any significant recovery of function.

It has been suggested, however, that presynaptic adaptations are not sufficient to mediate recovery of function. For example, Altar et al. (1987) reported that some presynaptic adaptations to a 6-OHDA lesion (metabolism and biosynthesis) do not account for the protracted time course of recovery of sensorimotor function. The ratio of DOPAC or HVA/DA and the rate of DA synthesis were just as elevated $3 \mathrm{~d}$ after a unilateral 6-OHDA lesion as at $18 \mathrm{~d}$, although the animals showed considerable recovery of function over this time period. Of course, neither the measure of DA metabolism nor the measure of biosynthesis is a reliable indicator of DA release or the extracellular concentration of DA (Commissiong, 1985; Westerink, 1985; Wood and Altar, 1988), and this may account for their poor relationship to behavior. Altar et al. (1987) also measured the postmortem tissue concentration of 3-methoxytyramine (3-MT) as an index of DA release, and again the ratio of 3-MT/DA was equally elevated at 3 and $18 \mathrm{~d}$ following the 6-OHDA lesion. But 3-MT may not be a good indicator of extracellular DA concentrations either. There is considerable evidence that 3-MT is a good index of DA release in intact animals (Wood and Altar, 1988; although see Vulto et al., 1988) but it may not reflect the extracellular concentration of $D A$, which is due, of course, to alternations in DA release, DA degradation, and DA reuptake. Measures of 3-MT in postmortem tissue may not reflect changes in the diffusibility of DA that occur sccondary to the loss of reuptake sites produced by a 6 -OHDA lesion. To determine whether the normalization of extracellular DA described here can account for the time course of behavioral recovery will require experiments in which both are measured directly.

It is interesting that after a lesion exceeded $90-94 \%$ very small percentage increments in lesion size had a disproportionate effect on the ability to maintain extracellular DA, as seen in the very sharp decline in extracellular DA around the $95 \%$ mark. The "collapse" of the system following a $>95 \%$ lesion may be related to an increased propensity for the remaining DA neurons to develop depolarization block (Hollerman and Grace, 1989). However, even in animals with a $>95 \%$ lesion, the decrease in the extracellular concentration of DA $(79.5 \%)$ was not as great as predicted by the decrease in the postmortem tissue concentration of DA (98.3\%).

Another important issue, especially as lesion size approaches or exceeds $95 \%$, is the amount of individual variation in the extracellular concentration of DA. Although animals with a $>95 \%$ lesion showed a marked decline in extracellular DA, there was considerable variation in the magnitude of the decline. It will be important to determine if variation in the ability to maintain extracellular DA following a lesion in this "critical zone" (90-95\%) is related to well-documented individual variation in the rate and extent of behavioral recovery (Marshall, 1979; Kozlowski and Marshall, 1981; Stricker and Zigmond, 1986).

Although animals with a $>95 \%$ lesion were impaired in their behavioral response to the amphetamine challenge, relative to controls, they did show a significant increase in locomotion and rearing. This occurred despite an almost complete failure to increase the extracellular concentration of DA in the striatum. The nucleus accumbens was not depleted of DA to nearly the same extent as the striatum, however (Table 1), and given the well-documented role of the nucleus accumbens in amphetamine-induced locomotion (Kelly et al., 1975), it is possible that the remaining DA terminals in the nucleus accumbens were sufficient to mediate the increase in behavioral activity observed here. In animals with an 80-95\% lesion the amphetamine challenge actually produced a significantly greater behavioral response to amphetamine than in controls. This occurred even though the amphetamine challenge increased the extracellular concentration of DA to a slightly lesser degree than in controls. This may be related to the role of nucleus accumbens as a mediator for locomotion, since Jones et al. (1989) have demonstrated that rats with striatal lesions are hyperactive when given amphetamine. A lesion of this size, however, is known to produce DA receptor supersensitivity (Mishra et al., 1974; Creese et al., 1977; Neve et al., 1982) and therefore the enhanced behavioral response could also be due to normal extracellular levels of DA acting on a supersensitive receptor population. The latter interpretation is supported by the observation that animals with a $20-80 \%$ lesion, which would not produce appreciable DA receptor supersensitivity, failed to show a significantly enhanced behavioral response to amphetamine.

\section{References}

Altar CA, Marien MR (1989) Preservation of dopamine release in the denervated striatum. Neurosci Lett 96:329-334.

Altar CA, Marien MR, Marshall JF (1987) Time course of adaptations in dopamine biosynthesis, metabolism, and release following nigrostriatal lesions: implications for behavioral recovery from brain injury. J Neurochem 48:390-399.

Breese GR, Traylor TD (1971) Depletion of brain noradrenaline and dopamine by 6-hydroxydopamine. Br J Pharmacol 42:88-99.

Castañeda E, Whishaw IQ, Lermer L, Robinson TE (1990) Dopamine depletion in neonatal rats: effects on behavior and striatal dopamine release assessed by intracerebral microdialysis during adulthood. Brain Res 508:30-39.

Commissiong JW (1985) Monoamine metabolites: their relationship and lack of relationship to monoaminergic neuronal activity. Biochem Pharmacol 34:1127-1131.

Creese I, Burt DR, Snyder SH (1977) Dopamine receptor binding enhancement accompanies lesion-induced behavioral supersensitivity. Science 197:596-598.

Doucet G, Descarries L, Garcia S (1986) Quantification of the dopamine innervation in adult rat neostriatum. Neuroscience 19:427445.

Heffner TG, Hartman JA, Seiden LS (1980) A rapid method for the 
regional dissection of the rat brain. Pharmacol Biochem Behav 13: 453-456.

Heikkila RE, Hess A, Duvoisin RC. (1984) Dopaminergic neurotoxicity of 1-methyl-4-phenyl-1,2,5,6-tetrahydropyridine in mice. Science 224:1451-1453.

Hollerman JR, Grace AA (1988) Nigral DA cell recruitment as a compensatory mechanism. Soc Neurosci Abstr 14:1146.

Hollerman JR, Grace AA (1989) Acute haloperidol administration induces depolarization block or nigral dopamine neurons in rats after partial dopamine lesions. Neurosci Lett 96:82-88.

Hollerman JR, Berger TW, Grace AA (1986) Compensatory changes in the activity of nigral dopamine cells in response to partial dopamine-depleting brain lesions. Soc Neurosci Abstr 12:872.

Hornykiewicz O (1972) Dopamine and extrapyramidal motor function and dysfunction. Res Publ Assoc Res Nerv Ment Dis 50:390415 .

Jones GH, Mittleman G, Rohbins TW (1989) Attenuation of amphetamine-stereotypy by mesostriatal dopamine depletion enhances plasma corticosterone: implications for stereotypy as a coping response. Behav Neurol Biol 51:80-91.

Kelly PH, Seviour PW, Iversen SD (1975) Amphetamine and apomorphine responses in the rat following 6-OHDA lesions of the nucleus accumbens septi and corpus striatum. Brain Res 94:507-522.

Kozlowski MR, Marshall JF (1981) Plasticity of neostriatal metabolic activity and behavioral recovery from nigrostriatal injury. Exp Neurol $74: 318-323$

Marshall JF (1979) Somatosensory inattention after dopamine-depleting intracerebral 6-OHDA injections: spontaneous recovery and pharmacological control. Brain Res 177:311-324.

Marshall JF, Teitelbaum P (1974) Further analysis of sensory inattention following lateral hypothalamic damage in rats. J Comp Physiol Psychol 86:375-395.

Marshall JF, Richardson JS, Teitelbaum P (1974) Nigrostriatal bundle damage and the lateral hypothalamic syndrome. J Comp Physiol Psychol 87:808-830.

Mishra RK, Gardner EL, Katzman R, Makman MH (1974) Enhancement o[ dopamine-stimulated adenylate cyclase activity in rat caudate after lesions in substantia nigra: evidence for denervation supersensitivity. Proc Natl Acad Sci USA; 71:3883-3887.

Neve KA, Kozlowski MR, Marshall JF (1982) Plasticity of neostriatal dopamine receptors after nigrostriatal injury: relationship to recovery of sensorimotor functions and behavioral supersensitivity. Brain Res $244: 33-44$.

Onn SP, Berger TW, Stricker EM, Zigmond MJ (1986) Effects of intraventricular 6-hydroxydopamine on the dopaminergic innervation of striatum: histochemical and neurochemical analysis. Brain Res 376:8-19.

Ranje C, Ungerstedt U (1977) High correlations between number of dopamine cells, dopamine levels and motor performance. Brain Res 134:83-93

Robinson TE, Whishaw IQ (1974) Effects of posterior hypothalamic lesions on voluntary behavior and hippocampal electroencephalograms in the rat. J Comp Physiol Psychol 86:768-786.

Robinson TE, Whishaw IQ (1987) Striatal dopamine release assessed with microdialysis following unilateral nigrostriatal damage. Soc Neurosci Abstr 13:218.

Robinson TE, Whishaw IQ (1988) Normalization of extracellular dopamine in striatum following recovery from a partial unilateral 6-OHDA lesion of the substantia nigra: a microdialysis study in freely moving rat. Brain Res 450:209-224.

Robinson TE, Becker JB, Young EA, Akil H, Castañeda E (1987) The effects of footshock stress on regional brain dopamine metabolism and pituitary beta-endorphin release in rats previously sensitized to amphetamine. Neuropharmacology 26:679-691.

Robinson TE, Jurson PA, Bennett JA, Bentgen KM (1988) Pcrsistent sensitization of dopamine neurotransmission in ventral striatum (nucleus accumbens) produced by past experience with (+)-amphetamine: a dialysis study in freely moving rat. Brain Res 462:211-222.

Robinson TE, Yew J, Paulson PE, Camp DM (1990) The long-term effects of neurotoxic doses of methamphetamine on the extracellular concentration of dopamine measured with microdialysis in striatum. Neurosci Lett 110:193-198.
Schallert T, Whishaw IQ (1978) Two types of aphagia and two types of sensorimotor impairment after lateral hypothalamic lesions: observations in normal weight, dieted, and fattened rats. J Comp Physiol Psychol 92:720-741.

Schallert T, Whishaw IQ, Ramirez VD, Teitelbaum P (1978) Compulsive, abnormal walking caused by anticholinergics in akinetic, 6-hydroxydopamine-treated rats. Science 199:1461-1463.

Schultz W (1982) Depletion of dopamine in the striatum as an experimental model of Parkinsonism: direct effects and adaptive mechanisms. Prog Neurobiol 18:121-166.

Stachowiak MK, Keller RJ, Stricker EM, Zigmond MJ (1987) Increased dopamine efflux from striatal slices during development and after nigrostriatal bundle damage. J Neurosci 7:1648-1654.

Stricker EM, Zigmond MJ (1974) Effects on homeostasis of intraventricular injections of 6-hydroxydopamine in rats. J Comp Physiol Psychol 86:973-994.

Stricker EM, Zigmond MJ (1976) Recovery of function after damage to central catecholamine-containing neurons: a neurochemical model for the lateral hypothalamic syndrome. In: Progress in psychobiology and physiological psychology (Sprague JM, Epstein A, eds), pp 121 187, New York: Academic.

Stricker EM, Zigmond MJ (1986) Brain monoamines, homeostasis and adaptive behavior. In: Handbook of physiology, Vol. 4, Intrinsic regulatory systems of the brain, pp 677-698, Bethesda, MD: American Physiological Society.

Teitelbaum P, Epstein AN (1962) The lateral hypothalamic syndrome: recovery of feeding and drinking after lateral hypothalamic lesions. Psychol Rev 69:74-90.

Ungerstedt $U$ (1968) 6-Hydroxy-dopamine induced degeneration of central monoamine neurons. Eur J Pharmacol 5:107-110.

Ungerstedt U (1971) Adipsia and aphagia after 6-hydroxydopamine induced degeneration of the nigro-striatal dopamine system. Acta Physiol Scand 367 (Suppl):95-122.

Vulto AG, Sharp T, Ungerstedt U, Versteeg DH (1988) Rapid postmortem increase in extracellular dopamine in the rat brain as assessed by brain microdialysis. J Neurochem 51:746-749.

Westerink BHC (1985) Sequence and significance of dopamine metabolism in the rat brain. Neurochem Int 7:221-227.

Whishaw IQ, Dunnett SB (1985) Dopamine depletion, stimulation or blockade in the rat disrupts spatial navigation and locomotion dependent upon beacon or distal cues. Brain Res 18:11-29.

Whishaw IQ, Robinson TE, Schallert T, De Ryck M, Ramirez VD (1978) Electrical activity of the hippocampus and neocortex in rats depleted of brain dopamine and norepinephrine: relations to behavior and effects of atropine. Exp Neurol 62:748-767.

Whishaw IQ, O'Connor WT, Dunnett SB (1986) The contributions of motor cortex, nigrostriatal dopamine and caudate-putamen to skilled forelimb use in the rat. Brain 109:805-843.

Wolf ME, Zigmond MJ, Kapatos G (1989) Tyrosine hydroxylase content of residual dopamine terminals following 6-hydroxydopamine administration: a flow cytometric study. J Neurochem 53:879-885.

Wood PL, Altar CA (1988) Dopamine release in vivo from nigrostriatal, mesolimbic, and mesocortical neurons: utility of 3-methoxytyramine measurements. Pharmacol Rev 40:163-187.

Zhang WQ, Tilson HA, Nanry KP, Hudson PM, Hong JS, Stachowiak MK (1988) Increased dopamine release from striata of rats after unilateral nigrostriatal bundle damage. Brain Res 461:335-342.

Zigmond MJ, Stricker EM (1972) Deficits in feeding behavior after intraventricular injection of 6-hydroxydopamine in rats. Science 177: 1211-1214.

Zigmond MJ, Stricker EM (1973) Recovery of feeding and drinking by rats after intraventricular 6-hydroxydopamine or lateral hypothalamic lesions. Science 182:717-720.

Zigmond MJ, Acheson AL, Stachowiak MK, Stricker EM (1984) Neurochemical compensation after nigrostriatal bundle injury in an animal model of preclinical parkinsonism. Arch Neurol 41:856-861.

Zigmond MJ, Stachowiak MK, Berger TW, Stricker EM (1986) Neurochemical events underlying continued function despite injury to monoaminergic systems. Exp Brain Res Suppl 13:119-128.

Zis AP, Fibiger HC, Phillips AG (1974) Reversal by L-dopa of impaired learning due to destruction of the dopaminergic nigro-neostriatal projection. Science 185:960-962. 